\title{
Performance of 3D Printing in Construction by Using Computer Control Technology
}

\author{
Zibo Zuo ${ }^{12}$ \\ Shanghai Construction Group Co., Ltd. \\ Shanghai, 200080, China \\ E-mail: zuozibo@foxmail.com \\ Jian Gong ${ }^{3}$ \\ Shanghai Construction Group Co., Ltd. \\ Shanghai, 200080, China \\ E-mail: gongjianescgtc.com.cn

\section{Yulin Huang} \\ Shanghai Construction Group Co., Ltd. \\ Shanghai, 200080, China \\ E-mail: hyl0409080163.com
}

\begin{abstract}
It is necessary to study a computer control technology to achieve the optimal balance of efficiency and accuracy of automation construction in the large-scale additive manufacturing processes. This paper proposes the mathematical models as a simulation tool and several printing parameters involving the performance capability for full-scale construction by using 3D printing. An approach of interpreting the printing accuracy is presented and the primary monitoring indexes based on computer technology are introduced to achieve high precision construction. A model of path planning and the optimization path generating algorithms under different conditions for the single nozzle system are investigated to minimize the printing duration. The research on the path planning of construction 3D printing for multiple nozzles and simultaneous multi-equipment based on artificial intelligent algorithm is prospected. The proposed technique provides a link for the integration of computer science technology and construction technology.
\end{abstract}

CENet 2017

22-23 July, 2017

Shanghai, China

${ }^{1}$ Speaker

${ }^{2}$ Correspongding Author

${ }^{3}$ This research is financially supported by National Key R\&D Plan (Project No. 2017YFC0805500 ) and Shanghai Construction Group Co., Ltd. (Project No. 14GLXX05\&16JCYJ-02) 


\section{Introduction}

The Rapid prototyping (RP) is a group of emerging techniques for quickly fabricating physical parts, assemblies or objects by directly using 3D computer aided design (CAD) data. $3 \mathrm{D}$ printing (3DP), also known as additive manufacturing ( $\mathrm{AM}$ ), refers to an RP processes to create a 3D object in which many thin layers of materials are formed under computer control to create an object [1].

The traditional construction industry still features intense labourers and craft orientation, which has resulted in several serious problems in the field of productivity, quality, safety, skilled labor shortages and waste produced. In order to address these issues and push construction towards automation, 3DP technology by using the building materials (sand, plaster, concrete, cementbased material, metal, ceramic and wood, etc.) based on the building information model (BIM), named construction 3D printing (C3DP), has been proposed and implemented in the field of construction and building. Researches and practices in terms of C3DP in the public domain are limited to three processes (Fig.1), namely: the contour crafting (invented by Behrokh Khoshnevis of the University of Southern California, US) [2], the D-Shape (invented by Enrico Dini of Monolite UK Ltd) [3] and the concrete printing (invented by Richard Buswell of Loughborough University, UK) [4]. The processes have verified the successful manufacturing of components of significant size and been suitable to construction and architectural applications. Generally speaking, the construction components are likely to be large volumes of material and large scale (even more than a few hundred meters) AM processes targeted at construction and architecture in comparison with the typical 3DP. High precision design requirements (accuracy requires millimeter or sub-millimeter) for C3DP, nevertheless, is difficult due to complex macro-structure of the tiny texture geometry because it needs to be compatible with efficient construction of large size buildings. It means that it is necessary to study a computer control technology (CCT) to achieve the optimal balance of efficiency and accuracy in construction. Currently, the problem is application specific by using C3DP. At present, few attempts have been reported in literature.

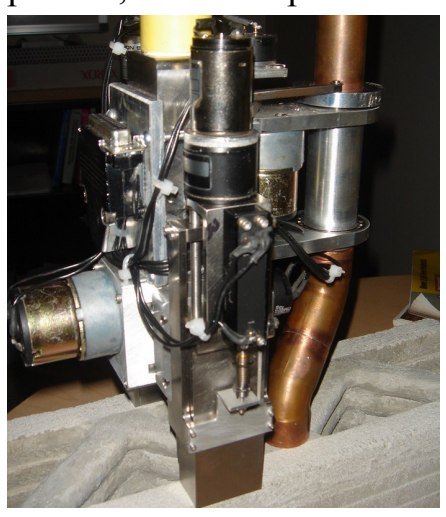

(a) Contour Crafting [2]

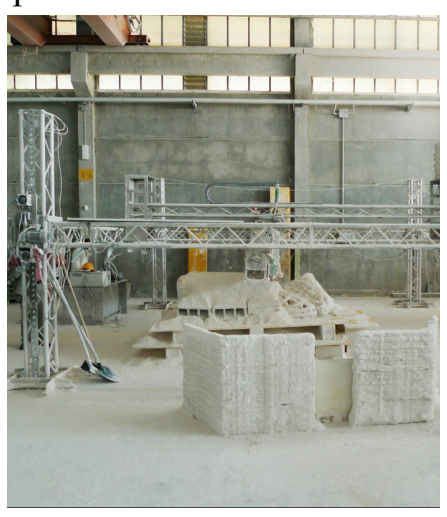

(b) D-shape [3]

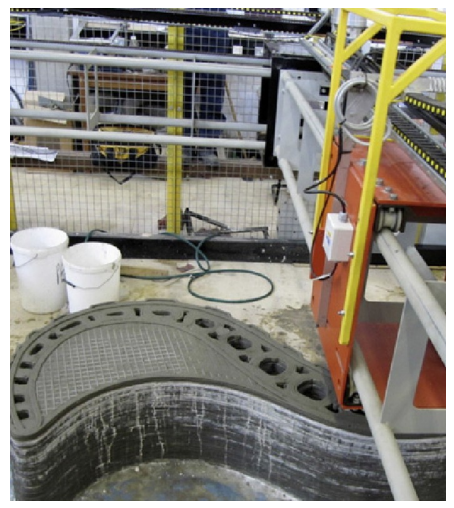

(c) Concrete Printing [4]

Figure 1: Examples of Full Scale Builds from C3DP process.

This paper proposes the mathematical models as a simulation tool and several printing parameters involving performance capability for full-scale construction by using 3D printing. In order to achieve high precision construction by using 3DP, an approach of interpreting printing accuracy is presented and the primary monitoring indexes based on computer technology are introduced. The methods of the optimization path planning by using CCT under different conditions are investigated to control the position of the printer head efficiently. 


\section{Mathematical Models of C3DP}

The mathematical models for large scale C3DP have been proposed, as illustrated in Fig. 2. As C3DP is an AM process as opposed to a subtractive, forming or phase change processes, the material is added as droplets ejected from a nozzle with the printer head moving freely along the $\mathrm{X}, \mathrm{Y}$ and $\mathrm{Z}$ axis. The size of a resulting primitive, as called the primitive element (PE), corresponds to the powder-binder agglomerate formed by several droplets (Figure 2(b)). A droplet called unit cell (UC) can be approximated to be a ball primitive in this method (Fig. 2(c)). With the nozzle ejects the printing material, a printing layer is formed by several PEs and then the construction component (CC) is fabricated.

Several printing parameters involving the performance capability of C3DP illustrated in Fig. 2 are shown as follows: $v_{x}, v_{y}$ and $v_{z}$ are the motion speed in the $\mathrm{X}, \mathrm{Y}$ and $\mathrm{Z}$ axis (usually $v_{Y}$ is smaller than $v_{X}$ ); $L_{x}, L_{y}$, and $L_{z}$ refer to the length, width and thickness of CC respectively; $D_{x}$, $D_{y}$ and $D_{z}$, determined by the factors including the UC diameter and the spacing increment, the flow rate of printing materials $V_{H}$, the motion speed of the nozzle and aperture of the nozzle $R_{H}$, etc., are the length, width and thickness of PE, while $D_{z}, D_{y}$ and $D_{x}$ refer to the layer thickness, the printing row width and the unit length of printing segment respectively in essence; $R$ is the diameter of UC and the value may be determined by various factors including UC chemical composition and size, powder chemical composition and size, powder bed density and surface tension of the UC; $R_{x x}, R_{y y}$ and $R_{z z}$ are the spacing increment of $\mathrm{UC}$ along $\mathrm{X}, \mathrm{Y}$ and $\mathrm{Z}$ axis.

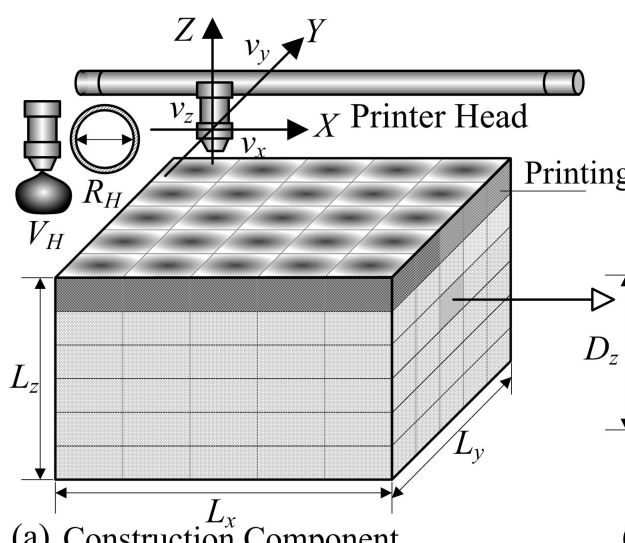

(a) Construction Component

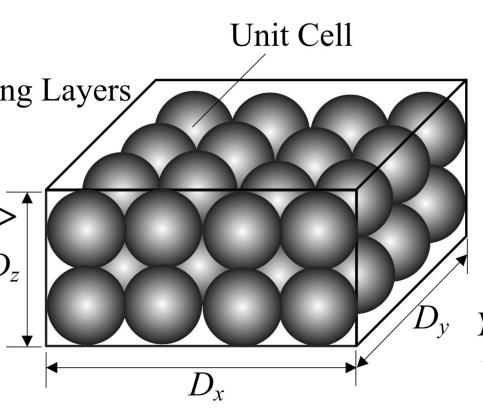

(b) Primitive Element

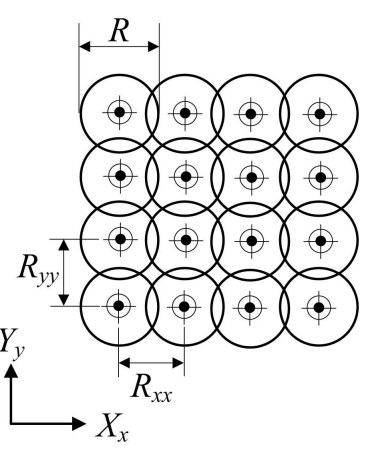

(c) Unit Cell

Figure 2: Mathematical Models of C3DP.

It should be noted that the preceding parameters will only be limited to the part geometry and the least common denominator involving process performance capability of all available C3DP techniques. The proposed mathematical models can be a simulation tool according to the C3DP process rules. However, the proposed models in this paper can't cover all issues in the C3DP such as material, surface property, dimensional tolerance and mechanical properties. Some new simulation tools [5] combined with the proposed models demand further studied.

\section{Printing Accuracy of C3DP by Using CCT}

The printing accuracy of C3DP equipment have to be considered as a contributor to the performance of C3DP techniques of a printed physical construction component from a designed BIM model. It is defined as the maximum deviation of PE including geometric deviation and position deviation as shown in Fig. 3. If there is a discrepancy in geometry between the printed 
component and the designed CAD model, the actual sizes $D_{x}, D_{y}, D_{z}$ of PE for the printed component along the $\mathrm{X}, \mathrm{Y}$ and $\mathrm{Z}$ axis can be defined as:

$$
\left\{\begin{array}{c}
D_{x}=R+m_{x x} R_{x x} \pm n_{x x} E_{x x} \\
D_{y}=R+m_{y y} R_{y y} \pm n_{y y} E_{y y} \\
D_{z}=R+m_{z z} R_{z z}+n_{z z} E_{z z}
\end{array}\right.
$$

where $m_{x x}, m_{y y}$ and $m_{z z}$ refer to the integer number of increment of UC along the X, Y and $\mathrm{Z}$ axis; $E_{x x}, E_{y y}$ and $E_{z z}$ refer to the maximum geometric deviation error of UC along the X, Y and $\mathrm{Z}$ axis; $n_{x x}, n_{y y}, n_{z z}=0$ (if $m_{x x}, m_{y y}, m_{z z}=0$ ); $n_{x x}, n_{y y}, n_{z z}=2$ (if $m_{x x}, m_{y y}, m_{z z} \neq 0$ ).

If there is a discrepancy in position between the printed component and the designed CAD model, the maximum position deviation $E_{x}, E_{y}$ and $E_{z}$ of $\mathrm{PE}$ for the printed component along the $\mathrm{X}, \mathrm{Y}$ and $\mathrm{Z}$ axis can be defined in Fig. 3(b). The values of $E_{x}, E_{y}$ and $E_{z}$ are the square root of the absolute value of the deviation error between the centroid coordinates of the printed component and the designed CAD model.

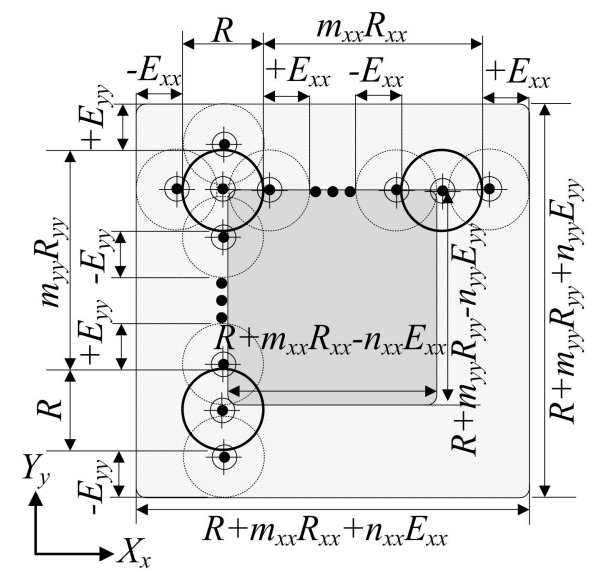

(a) Geometric deviation

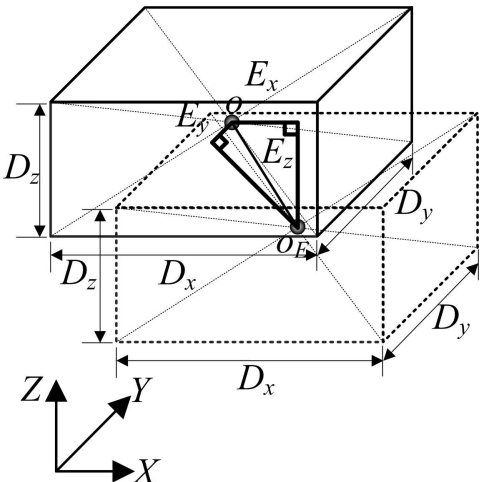

(b) Position deviation

Figure 3:Printing Accuracy for C3DP Process.

The proposed maximum deviation in this paper can reflect the printing accuracy to explain any unpredictable between the printed component and the designed CAD model. In order to achieve high precision construction by using 3DP, it is necessary to use real-time monitoring and CCT to monitor the changes in $E_{x x}, E_{y y}, E_{z z}, E_{x}, E_{y}$ and $E_{z}$. Moreover, it is important to study the techniques of printing errors prediction and control. The final combination of the equipment errors, including the controller errors, can be made within an allowable error.

\section{Optimization path planning of C3DP using CCT}

\subsection{Problem Statement}

The printing speed mainly depends on both the motion speed $\left(v_{x}, v_{y}\right.$, and $\left.v_{z}\right)$ and the path of the printer head [6]. In fact, once $D_{x}, D_{y}$ and $D_{z}$ give the design value, the motion speed ( $v_{x}, v_{y}$, and $v_{z}$ ) is determined. In order to minimize the printing duration, it is necessary to carry out the optimization of the path planning using CCT to control the position of the printer head efficiently. Therefore, the optimization path planning will also have to be considered as a contributor to the performance of C3DP techniques.

Fig. 4 shows one path planning description of C3DP process of a construction component. The nozzle sprays printing material from $P_{1}$ to $S_{1}$, and then moves to $S_{2}$ without spraying. It 
sprays again when moving from $S_{2}$ to $P_{2}$ to complete the second path. This process is repeated until the nozzle sprays from $P_{f}$ to $S_{e}$ to finish one layer, and the layering process is repeated until the construction component is printed. There are thousands of such paths illustrated above. So the main goal is to generate the shortest path from a predefined start point to a predefined end point which fulfils the whole printing area. The model of the path planning can be stated as follows.

The printing layer as a set of the binary points [7] (an example in Fig. 4):

$$
P_{i j}=\left\{\begin{array}{c}
1 \text { if printing point } \\
0 \text { otherwise }
\end{array} \quad i \in L_{x}, j \in L_{y}\right.
$$

The length between two points is defined as

$$
L\left(P_{i j}, P_{(i+1 j+1)}\right)=\sqrt{\left(x_{i}-x_{(i+1)}\right)^{2}-\left(y_{i}-y_{(i+1)}\right)^{2}}
$$

To find the final path $F=\left[P_{1}, P_{2}, \ldots P_{f}, S_{1}, S_{2}, \ldots S_{e}\right]$, it is necessary to minimize the total length of the found path $L_{F}$ as

$$
\begin{gathered}
\min L_{F}=\min \left\{L_{P}+L_{S}\right\}=\min \left\{\sum_{i=1}^{f} \sum_{j=1}^{e} \sqrt{\left(x_{P i}-x_{S j}\right)^{2}-\left(y_{P i}-y_{S j}\right)^{2}}\right. \\
\left.+\sum_{i=1}^{e-2} \sqrt{\left(x_{S i}-x_{(S i+1)}\right)^{2}-\left(y_{S i}-y_{(S i+1)}\right)^{2}}+\sum_{j=2}^{f-1} \sqrt{\left(x_{P i}-x_{(P i+1)}\right)^{2}-\left(y_{P i}-y_{(P i+1)}\right)^{2}}\right\}
\end{gathered}
$$

Where $L_{P}$ is the sum of the length between the points in the sub-paths (Such as $P_{1} S_{1}$, $\left.S_{2} P_{2} \ldots P_{f} S_{e}\right) ; L_{S}$ is the length between the last point in the one sub-path and the first point in the next sub-path (Such as $\left.S_{1} S_{2}, P_{2} P_{3} \ldots S_{e-2} S_{e-1}, P_{f-1} P_{f}\right) ; 0 \leq(f+e) \leq A, A$ is the total number of subpaths; $\left(x_{i}, y_{i}\right)$ is the position coordinate of point $P_{i j} ;\left(x_{P i}, y_{P i}\right)$ and $\left(x_{S i}, y_{S i}\right)$ are the position coordinate of points $P_{i}$ and $S_{j}$; it is subject to the constraint that each point can be visited only once.

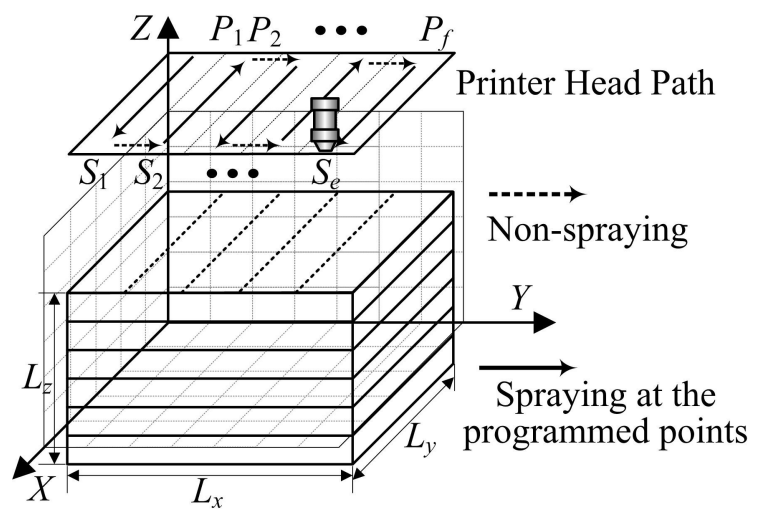

Figure 4: Path Planning Description of C3DP Process.

\subsection{Path Planning Algorithms and Case Study}

We have developed a C3DP equipment that can be used for both underground structures and building construction, as shown in Fig.5. The underground structure needs to be constructed after excavation has been completed. The equipment can be considered as a C3DP equipment for the single nozzle system. The approach of finding the optimal tool path is to solve the above model problem iin Eq. (4.3) by using a reasonable algorithm. 


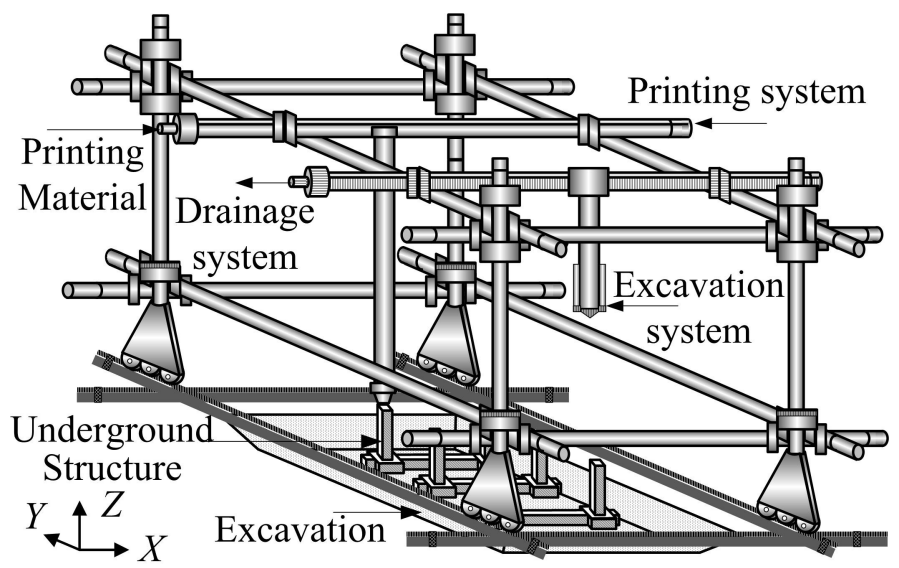

Figure 5:C3DP Equipment for the Single Nozzle System.

The traditional algorithm (Z-type), the Zigzag algorithm and the contour algorithm have been adopted to find the acceptable result within reasonable time limit. A set of simulations have been performed with printed underground structure. The simulation results are illustrated in Table 1. The optimal paths are obtained finally as illustrated in Fig. 6. It can be observed that the optimal paths are different depending on the algorithm. The Zigzag algorithm for the single nozzle system presented here seems to be better than the rest of algorithms. It should be pointed out that each algorithm has its own limitations. With the development of multiple nozzles, simultaneous multi-equipment [8], adaptive expansion equipment (Fig.5), ultra-high complexity construction equipment, etc., the traditional algorithms are no longer suitable to the optimization path planning of C3DP. Research on the new algorithms such as artificial intelligence algorithms for path planning may be the future research direction.

\begin{tabular}{ccc}
\hline Algorithm & Path Length $(\mathrm{m})$ & Length of Transition Paths $(\mathrm{m})$ \\
\hline Traditional (Z-type) & 346.3246 & 102.3246 \\
Contour & 257.9149 & 20.9443 \\
ZigZag & 239.3137 & 0 \\
\hline
\end{tabular}

Table 1: Simulation Results.

\section{Conclusion}

This research was intended to analyze the performance of 3DP in automation construction by using CCT in order to achieve the optimal balance of efficiency and accuracy of the construction in large scale additive manufacturing processes.

Mathematical models as a simulation tool and several printing parameters involving performance capability for full-scale construction by using 3DP have been proposed. An approach of interpreting the printing accuracy has been presented and the primary monitoring indexes based on computer technology have been introduced to achieve high precision construction. A model of the path planning and the optimization path generating algorithms for the single nozzle system have been investigated to minimize the printing duration. The research on the path planning of C3DP for multiple nozzles, simultaneous multi-equipment and adaptive expansion equipment based on artificial intelligent algorithm is prospected. The proposed technique provides a link for the integration of computer science technology and construction technology. 
This paper focuses on some new ideas for the application of computer control technologies in construction by using 3DP. The research of the fine monitoring method of printing accuracy and optimal path planning algorithms in practical applications need to be further studied.

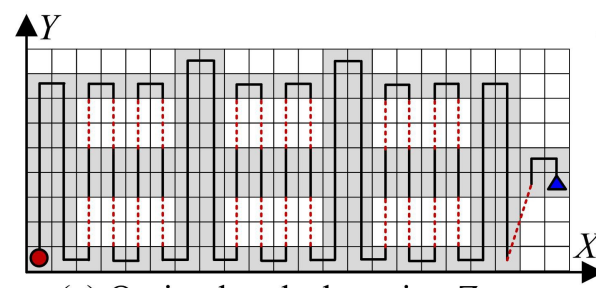

$Y$

(a) Optimal paths by using Z-type

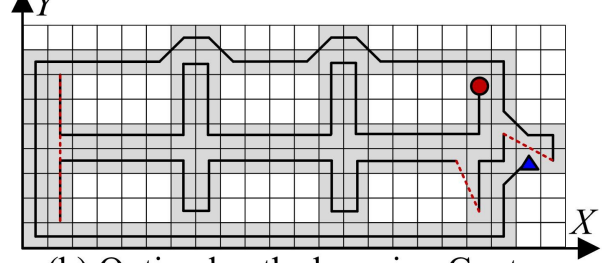

(b) Optimal paths by using Contour

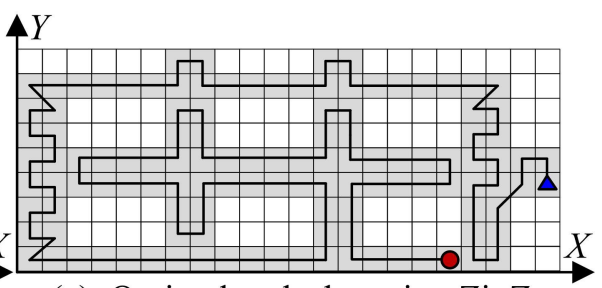

(c) Optimal paths by using ZigZag

Figure 6: Examples of Path Planning.

\section{References}

[1]H.J. Jeea, E. Sachsb, A visual simulation technique for 3D printing[J]. Advances in Engineering Software, 2000, 31: 97-106.

[2]B. Khoshnevis, Contour Crafting-state of development[C]. Solid Freeform Fabrication Proceedings 1999, Texas, USA, Aug, 1999: 743-750.

[3]G. Cesarettia, E. Dinib, X. D. Kestelierc, V. Collad, L. Pambaguiane, Building components for an outpost on the Lunar soil by means of a novel 3D printing technology[J]. Acta Astronautica, 2014, 93: 430-450.

[4]S. Lim, T. Le, J. Webster, R. Buswell, S. Austin, A. Gibb, T. Thorpe, Fabricating construction components using layer manufacturing technology[C]. Global Innovation in Construction Conference 2009 (GICC’09), Leicestershire, UK, Sep, 2009, 13-16.

[5]A. Qureshi, B. Li, K. T. Tan, Numerical investigation of band gaps in 3D printed cantileverinmass metamaterials[J]. Scientific Reports, 2016, 6:28314-1-28314-10.

[6]N. Ganganath, C.T. Cheng, K. Y. Fok, K. T. Chi, Trajectory planning for 3D printing: a revisit to traveling salesman problem [C]. Control, Automation and Robotics (ICCAR), 2016 2nd International Conference on, Hong Kong, China, Apr, 2016: 1-4.

[7]M.Wojcik, I. Pozniak-Koszalka, L. Koszalka, A. Kasprzak, Advances in Applied Digital Human Modeling and Simulation[M]. Springer, Berlin, Germany, pp, 291-302 (2017).

[8]J. Zhang, B. Khoshnevis, Optimal machine operation planning for construction by Contour Crafting[J]. Automation in Construction, 2013, 29:50-67. 